\section{Quantum and Classical Game Strategies}

A recent paper [1] discusses how quantum mechanics affects game theory, with the conclusion that for certain games a suitable quantum strategy is able to beat any classical strategy. That conclusion is correct. Quantummechanical protocols are known to be superior to classical protocols both in solving certain computational tasks and for cryptographic purposes, both of which can be reformulated in terms of games [1]. The aim of this Comment, however, is to point out that the particular example presented in [1] to prove that quantum strategies are superior to classical strategies is flawed. There is nothing quantum mechanical about that strategy because it can be implemented classically, as will be shown below. Moreover, the example shows only that a player using a restricted set of strategies can be beaten by a player who uses an extended set of strategies. But this is unsurprising.

The game is penny flipping, where player $P$ places a penny head up in a box, after which player $Q$, then player $P$, and finally player $Q$ again, can choose to flip the coin or not, but without being able to see it. If the coin ends up being head up, player $Q$ wins, else player $P$ wins. The winning (or cheating, depending upon one's perspective) quantum strategy of $Q$ now consists of putting the penny into a superposition of head up and down. Since player $P$ is allowed to interchange only up and down he is obviously not able to change that superposition, so that $Q$ wins the game by rotating the penny back to its initial state.

It is easy to see why $Q$ 's "quantum" strategy, when applied to a two-state quantum system, can be implemented classically. First of all, one implementation of the game could make use of photon polarization, with, for example, the right- and left-hand circular polarizations corresponding to heads and tails, according to

$$
\begin{aligned}
\vec{e}_{\text {heads }} & =\left(\vec{e}_{x}+i \vec{e}_{y}\right) / \sqrt{2}, \\
\vec{e}_{\text {tails }} & =\left(\vec{e}_{x}-i \vec{e}_{y}\right) / \sqrt{2} .
\end{aligned}
$$

In spite of the use of the word photon, this experiment can just as well be performed with classical light. It just so happens that classical transformations of the polarization vector can be represented by unitary operators. For instance, imagine that flipping the coin is implemented by sending the light through a birefringent medium that shifts the phase of the $y$ component of the polarization vector by $\pi$, while leaving the $x$ component unchanged. Then it is clear that player $Q$ indeed wins by changing the polar- ization state into a superposition of the two circular polarizations, as this just means polarizing the light in the $\vec{e}_{x}$ direction, which will be unaffected by $P$ 's action.

More poignantly, a similar strategy can be devised for a classical penny. Flipping the penny can be implemented by rotating the penny around a fixed axis in the horizontal plane on which the coin is initially lying. Player $Q$ can now win (or cheat, depending upon one's perspective) by putting the penny on its edge with its plane normal to the rotation axis. The fact that the orientation of the coin might have changed in the end is an artifact of a penny having more degrees of freedom than are relevant for the game.

Finally, and perhaps most importantly, there is a very good reason why a game with a single qubit does not show any improvement of quantum strategies over classical strategies. A single qubit is not a truly quantum system in the sense that its dynamics and its response to measurements can all be mocked up by a classical hidden-variable model. There are no Bell inequalities [2] or a Kochen-Specker theorem [3] for a two-dimensional system that forbids the existence of a classical model. In fact, such a model can be constructed explicitly [4]. Of course, this does not mean that every quantum system can be mimicked classically. But the fact that more complicated games, involving more than a single qubit, are hard or even impossible to implement classically does not take away from the fact that Meyer's particular example is incorrect. In fact, countering the present objection by pointing out that it would have failed for larger systems is not unlike saying after a lost game of chess: "But if we would have played on a larger board, I would have won."

I thank Chris Fuchs for useful discussions. This work was funded by DARPA through the QUIC (Quantum Information and Computing) program administered by the U.S. Army Research office, the National Science Foundation, and the Office of Naval Research.

\section{S. J. van Enk}

Norman Bridge Laboratory of Physics

California Institute of Technology 12-33

Pasadena, California 91125

Received 1 February 1999

PACS numbers: 03.67.-a, 02.50.Le, 03.65. $-\mathrm{w}, 89.80 .+\mathrm{h}$

[1] D. Meyer, Phys. Rev. Lett. 82, 1052 (1999).

[2] J. S. Bell, Rev. Mod. Phys. 38, 447 (1966).

[3] S. Kochen and E. P. Specker, J. Math. Mech. 17, 59 (1967).

[4] J.F. Clauser, Am. J. Phys. 39, 1095 (1971). 